\title{
Modeling age-based maintenance strategies with minimal repairs for systems subject to competing failure modes due to degradation and shocks
}

\author{
K.T. Huynh ${ }^{\mathrm{a}, *}$, I.T. Castro ${ }^{\mathrm{b}}$, A. Barros ${ }^{\mathrm{a}}$, C. Bérenguer ${ }^{\mathrm{a}}$ \\ ${ }^{a}$ Université de technologie de Troyes - Institut Charles Delaunay and STMR UMR CNRS 6279 - 12, rue Marie Curie, \\ BP2060, 10010 Troyes cedex, France \\ ${ }^{b}$ Departamento de Matemáticas. Escuela Politécnica. 10071 Cáceres, Spain
}

\begin{abstract}
This paper deals with maintenance strategies with minimal repairs for single-unit repairable systems which are subject to competing and dependent failures due to degradation and traumatic shocks. The main aims are to study different approaches for making a minimal repair decision (i.e. time-based or condition-based) which is a possible corrective maintenance action under the occurrence of shocks, and to show under a given situation which approach can lead to a greater saving in maintenance cost. Two age-based maintenance policies with age-based minimal repairs and degradation-based minimal repairs are modeled, and their performance is compared with a classical pure age-based replacement policy without minimal repairs.

Numerical results show the cost saving of the maintenance policies and allow us to make some conclusions about their performance under different situations of system characteristic and maintenance costs. It is shown that carrying out minimal repairs is useful in many situations to improve the performance of maintenance operations. Moreover, the comparison of optimal maintenance costs incurred by both maintenance policies with minimal repairs allows us to justify the appropriate conditions of time-based minimal repair approach and condition-based minimal approach.
\end{abstract}

\section{Keywords:}

Gamma process, non-homogeneous Poisson process, age replacement policy, minimal repairs, random inspection, dynamic environment

\section{Acronyms}

$(\tau, T)$ policy Age-based maintenance policy with minimal repairs depending on the system age

$(A, T)$ policy Age-based maintenance policy with minimal repairs depending on the system degradation PAR policy Pure (or classical) age-based replacement policy

\footnotetext{
* Corresponding author

Email addresses: tuan.huynh@utt.fr (K.T. Huynh), inmatorres@unex.es (I.T. Castro), anne.barros@utt.fr (A. Barros), christophe.berenguer@utt.fr (C. Bérenguer)
} 


\section{Introduction}

With the growth in complexity of modern systems, maintenance strategies have become more important and play an essential role directly related to the competitiveness of organizations. Many mechanical and structural systems (e.g. cutting tool [1], hydraulic structure [2], airplane engine compressor blades [3], corroding pipelines [4], etc.) usually suffer an underlying degradation process which can cause random failures. Such degradation process can be modeled using stochastic processes. According to Lehmann [5], the stochastic-processes-based approach shows great flexibility in describing the failure-generating mechanisms and can give alternative time-to-failure-distributions defined by the degradation model.

In the literature, developments on maintenance models based on the degradation process have provided satisfactory results for the maintenance operation (e.g. see $[6,7]$ for time-based maintenance model and [810] for condition-based maintenance model). But, considering a degradation process only, as in these papers, seems to be unsatisfactory for modeling dynamic systems that suffer a degradation with their operational age and that are subject to traumatic events or shocks which can lead to a sudden failure [11]. In these dynamic systems, the system is regarded as failed when its degradation reaches a critical threshold or when a catastrophic shock occurs although the degradation process has not reached the threshold. The models that describe these systems are called Degradation-Threshold-Shock (DTS) models. As far as we know, Lemoine and Wenocur were the first to analyze the DTS model [12]. Singpurwalla presents a comprehensive review of this class of models in [11]. Lehmann derived in [5] an expression of the survival function and the failure rate of the time to the system failure in the DTS model.

Most of the papers that deal with competing DTS models assume that the degradation process and the shock process are independent $[5,13-15]$. But, in many practical situations, the dependence between them is of importance and should not be neglected, as it leads to a competing risk model. Therefore, the present paper aims to expand the DTS model [5] by introducing the dependence between the shock process and the degradation process. The dependence between the shock process and the degradation process of the DTS model considered in this paper is described showing that the failure rate of the shocks depends on the degradation level of the system.

Based on such a degradation/failure model, this paper aims to develop a mathematical model to assess the benefit of carrying out minimal repairs as possible corrective maintenance actions under the occurrence of shocks. The basic concept of minimal repair was first introduced by Barlow and Hunter in [16], this has been extended in many ways, see e.g., [17-21]. The minimal repair is described by the fact that the repair action returns the system to an operational state but the system characteristics are the same as just before 
the failure. For a repairable system, carrying out minimal repairs is a natural approach, because it can keep the system working at a minimal cost. However, with a competing risk model under consideration as in this paper, performing a minimal repair requires more knowledge about the system state (e.g. failure type or degradation level) and cannot restore the system to "as good as new" condition as in a system replacement, hence it can incur an unnecessary maintenance cost. So the question is whether to replace the system or to perform a minimal repair when the system fails, and under which conditions the minimal repair can delay the undertaking of system replacements at a more convenient time. We analyze whether carrying out a minimal repair should be based on the operational age of the system (time-based decision) or should be based on the degradation of the system (condition-based decision). To answer the above questions, we develop the maintenance cost models for two age-based policies: a policy implementing age-based minimal repairs at failure times, a policy implementing degradation-based minimal repairs at failure times. These policies are compared with a pure age-based replacement policy without minimal repairs in order to assess the value of the minimal repairs. Furthermore, comparing optimal maintenance costs incurred by both kinds of maintenance policy with minimal repairs allows us to give some conclusions about their performance under different situations of system characteristics and maintenance costs.

Hence, the main aims and contributions of the present study are:

1. Expanding DTS models introducing a dependence scheme between the shock process and the degradation process.

2. Developing the analytical cost models for the age-based maintenance policies which use different approaches to make a minimal repair decision.

3. Performing a quantitative comparison between the proposed maintenance models to assess the value of the minimal repair in the maintenance.

4. Providing the indicators for choosing the adequate maintenance policy according to different system characteristics.

The remainder of this paper is organized as follows. Section 2 is devoted to modeling the different competing failure modes of the system and to characterizing the associated failure times. The detailed description and formulation of the different age-based maintenance policies with and without minimal repairs are introduced in Section 3. Then in Section 4, we give the comparison of the relative gain in the optimal maintenance cost rate between the maintenance strategies under the different possible situations to assess the effect of resorting to minimal repairs and the value of investing in condition monitoring. Finally, the paper ends with some conclusions and directions for future works. 


\section{System degradation and failure modeling}

The present paper considers a single-unit repairable system whose failures are due to the competing causes of degradation and shocks. The system is described by a so-called Degradation-Threshold-Shock (DTS) model [5]. In such a model, the degradation is modeled using a time-dependent stochastic process, and the system is regarded as failed when the degradation process reaches a critical threshold or when a shock occurs although the degradation process has not reached the threshold. As Singpurwalla advocated in [11], such a failure/degradation model can be seen as a combination and a more versatile - and hopefully realistic extension of many classical failure models based either only on degradation or only on parametric lifetime distributions. More recently, Bocchetti et al. apply this model to describe the competing risks due to wear degradation and thermal cracking for the cylinder liners of a marine Diesel engine [22]. In the following, the modeling of the different failure modes and the distributions of the associated hitting times are analyzed in detail, highlighting the dependence between the failure types.

\subsection{Degradation-based failure}

\subsubsection{Degradation modeling}

We consider a system deteriorating with use and age, and subject to a continuous accumulation of degradation. The degradation evolution of the system is modeled by a stochastic process. Let $X(t)$ be the accumulated degradation (or wear level) at time $t$. If no maintenance action is performed, the stochastic process $\{X(t), t \geq 0\}$ is continuous-time and monotonically increasing with $X(0)=0$. The degradation is strictly increasing which means that the system worsens with time due to ageing and accumulated wear or damage.

We assume in this paper that $\{X(t), t \geq 0\}$ is a homogeneous gamma process as defined by Singpurwalla in [39] (by opposition to the gamma process defined by Berman in [36]). According to this definition, the gamma process is a monotone increasing stochastic process with continuous state space (often used to model a continuous degradation phenomena), completely defined by the law of the increments between two arbitrary instants (usually named increments of degradation) in the state space. This law is a gamma law. In the framework of continuous state degradation models, this definition of the Gamma process is really mainly used [27]. The gamma processes were satisfactorily fitted to data of different gradual degradation phenomena such as erosion, corrosion, concrete creep, crack growth or wear of structural components [2325]. Moreover, the existence of an explicit probability distribution function of the gamma process permits feasible mathematical developments. Therefore, since the initial proposal by Abdel-Hameed in [26], the gamma process has been analyzed for different maintenance applications by several authors (see [27] for a thorough review on the use of gamma process in maintenance modeling).

The gamma process assumption for the degradation means that the probability density function of the degradation level $X(t)$ at time $t$ is a gamma density function with shape parameter $\alpha t$ and scale parameter 
$\beta$,

$$
f_{\alpha t, \beta}(u)=\frac{\beta^{\alpha t} u^{\alpha t-1} e^{-\beta u}}{\Gamma(\alpha t)}, \quad u \geq 0,
$$

where $\Gamma$ is the function gamma given by

$$
\Gamma(\alpha)=\int_{0}^{\infty} u^{\alpha-1} e^{-u} d u
$$

Such a process depends on two parameters $\alpha$ and $\beta$ which allow to model various degradation behaviors of the system from almost deterministic to highly variable. The parameters $\alpha$ and $\beta$ can be estimated from degradation data with classical statistical methods. The average degradation rate is $m=\alpha / \beta$ and its variance is $\sigma^{2}=\alpha / \beta^{2}$.

\subsubsection{Hitting times of the degradation process}

To characterize the degradation-based failures, it is necessary to study the hitting times of the gamma process. Two types of hitting times are showed in this section.

Firstly, we assume the system fails when the level of degradation exceeds a predetermined failure threshold $L$ which depends on the properties of the considered system. Denoting by $\sigma_{L}$ the time at which the degradation failure occurs

$$
\sigma_{L}=\inf \{t \geq 0, X(t) \geq L\}
$$

and by $F_{\sigma_{L}}$ its distribution, one obtains

$$
F_{\sigma_{L}}(t)=P\left[\sigma_{L} \leq t\right]=P[X(t) \geq L]=\int_{L}^{\infty} f_{\alpha t, \beta}(x) d x=\frac{\Gamma(\alpha t, L \beta)}{\Gamma(\alpha t)}, \quad t \geq 0,
$$

where

$$
\Gamma(\alpha, x)=\int_{x}^{\infty} z^{\alpha-1} e^{-z} d z
$$

denotes the incomplete gamma function for $x \geq 0$ and $\alpha>0$. Distribution $F_{\sigma_{L}}$ in (2) is known as the first hitting time distribution to the level $L$ with density function given by [27]

$$
f_{\sigma_{L}}(t)=\frac{\partial}{\partial t} F_{\sigma_{L}}(t)=\frac{\alpha}{\Gamma(\alpha t)} \int_{L \beta}^{\infty}\{\log (z)-\psi(\alpha t)\} z^{\alpha t-1} e^{-z} d z, \quad t \geq 0,
$$

where the function $\psi(a)$ is called the digamma function that corresponds to the derivative of the logarithm of the gamma function

$$
\psi(a)=\frac{\Gamma^{\prime}(a)}{\Gamma(a)}=\frac{\partial \log \Gamma(a)}{\partial a} .
$$

Let $M$ be a degradation level with $M<L$ and let $\sigma_{M}$ be the time in which the degradation of the system exceeds the value $M$ with distribution $F_{\sigma_{M}}$. In the models showed subsequently, the distribution of the random variable $\sigma_{L}-\sigma_{M}$ is used but it is more difficult to derive because of the "overshoot behavior" of the 
gamma processes [28], i.e., $X\left(\sigma_{M}\right) \neq M$ and $\sigma_{L}-\sigma_{M} \neq \sigma_{L-M}$. The distribution of $\sigma_{L}-\sigma_{M}$ was obtained by [9] and it is given by

$$
\bar{F}_{\sigma_{L}-\sigma_{M}}(t)=\left\{\begin{array}{cc}
-\iint_{M<x<L, 0<x+y<L, 0<y}\left(\int_{0}^{\infty} f_{\alpha u, \beta}(x) d u\right) \frac{\partial f_{\alpha t, \beta}(y)}{\partial t} d x d y & y>x>0 \\
\int_{t}^{\infty} \int_{0}^{y-t} \alpha \int_{0}^{M} f_{\alpha x, \beta}(w) d w\left(\int_{L-y}^{\infty} \frac{e^{-\beta z}}{z} d z\right) d x d y & y=x>0
\end{array}\right.
$$

Expression (4) is complicated and specially burdensome to compute numerically. This fact makes us propose an approximation of the distribution of the random variable $\sigma_{L}-\sigma_{M}$ to make the computation faster and easier. This approximation is based on the distribution of $\sigma_{L-M}$. If the trajectories of the process $\{X(t), t \geq 0\}$ were continuous, then $\sigma_{L}-\sigma_{M}$ would have the same probability as $\sigma_{L-M}$. But, since $\{X(t), t \geq 0\}$ is a jump process (see [27, 29] for more properties of gamma process), $X\left(\sigma_{M}\right) \geq M$ and hence $E\left[X\left(\sigma_{M}\right)\right] \geq M$. Bérenguer et al. proposed in [9] as approximation of $E\left[X\left(\sigma_{M}\right)\right]$ the following expression

$$
E\left[X\left(\sigma_{M}\right)\right]=\frac{\alpha}{\beta} E\left[\sigma_{M}\right]=\frac{\alpha}{\beta} \frac{1}{\alpha}\left(\beta M+\frac{1}{2}-\int_{\beta M}^{\infty}(\varphi(y)-1) d y\right),
$$

where $\varphi$ is a function closed to 1 ,

$$
\varphi(y)=\int_{0}^{\infty} f_{u, 1}(y) d u, \quad y \geq 1
$$

hence, when $\beta M$ is large enough (at least greater 1),

$$
E\left[X\left(\sigma_{M}\right)\right] \simeq M+\frac{1}{2 \beta} .
$$

Consequently, the distribution of the random variable $\sigma_{L}-\sigma_{M}$ can be approximated by the distribution of $\sigma_{L-M-1 /(2 \beta)}$ when $\beta M<\beta L-1 / 2$, thus the distribution of $\sigma_{L}-\sigma_{M}$ given in (4) and its density $f_{\sigma_{L}-\sigma_{M}}$ can be approximated as

$$
\begin{aligned}
F_{\sigma_{L}-\sigma_{M}}(t) & \simeq F_{\sigma_{L-M-\frac{1}{2 \beta}}}(t)=\frac{\Gamma(\alpha t, \beta(L-M)-1 / 2)}{\Gamma(\alpha t)} \\
f_{\sigma_{L}-\sigma_{M}}(t) & \simeq f_{\sigma_{L-M-\frac{1}{2 \beta}}}(t)=\frac{\alpha}{\Gamma(\alpha t)} \int_{(L \beta-M \beta-1 / 2)}^{\infty}\{\log (z)-\psi(\alpha t)\} z^{\alpha t-1} e^{-z} d z
\end{aligned}
$$

and this approximation will be used later in the numerical examples to compare different maintenance strategies.

\subsection{Shock failures}

\subsubsection{Shock failures modeling}

As mentioned above, in most practical situations the system failure is not solely due to degradation but also to shocks. Moreover the degradation process and the shock process can depend on each other, for example the higher the degradation, the more the system is vulnerable to shocks. We assume that the shock arrival times can be modeled by a non-homogeneous Poisson process $\left\{N_{s}(t), t \geq 0\right\}$ with stochastic increasing 
intensity which depends on the degradation level $r(X(t))$, and that can represented by the following relation [30]

$$
r(X(t))=r_{1}(t) 1_{\{X(t) \leq M\}}+r_{2}(t) 1_{\{X(t)>M\}}, \quad t \geq 0,
$$

where $1_{\{\}}$denotes the indicator function which equals 1 if the argument is true and 0 otherwise, $r_{1}(t)$ and $r_{2}(t)$ denote two continuous and non-decreasing failure rates at time $t$ with $r_{1}(t) \leq r_{2}(t), \forall t \geq 0$. The quantity $M$ represents a fixed degradation level. The quantities $r_{1}(t), r_{2}(t)$ and $M$ are the parameters of the shock process and can be estimated from shock failures and degradation data with classical statistical methods. Expression (5) means that the degradation evolution affects to the occurrence of shock failures, that is, the system is more prone to shock failures when the degradation increases and exceeds a given degradation level.

\subsubsection{Failure time of the shock process}

Let $N_{s}(t)$ be the number of shocks in $(0, t]$. Under this model, the failure rate for the shocks $r(X(t))$ given by (5) is stochastic and the process $\left\{N_{s}(t), t \geq 0\right\}$ is called a doubly stochastic process or a Cox process (see [31] for a theoretical development of the Cox process). We denote by $\bar{F}_{1}$ and $\bar{F}_{2}$ the survival functions associated with the failure rate functions $r_{1}$ and $r_{2}$, that is,

$$
\left.\bar{F}_{i}(t)=\exp \left\{-\int_{0}^{t} r_{i}(u) d u\right)\right\}, \quad t \geq 0, i=1,2 .
$$

Let $\sigma_{s}$ be the time to a failure provoked by a shock, that is,

$$
\sigma_{s}=\inf \left\{t \geq 0, N_{s}(t)=1\right\}
$$

and let $\bar{F}_{s}(t)$ be its survival function. One has

$$
\begin{aligned}
\bar{F}_{s}(t) & =P\left[\sigma_{s}>t\right] \\
& =P\left[\sigma_{s}>t \mid X(t) \leq M\right] P[X(t) \leq M]+P\left[\sigma_{s}>t, X(t)>M\right] \\
& =\bar{F}_{\sigma_{M}}(t) \bar{F}_{1}(t)+\int_{0}^{t} f_{\sigma_{M}}(u) \bar{F}_{1}(u) \exp \left\{-\int_{u}^{t} r_{2}(v) d v\right\} d u, \quad t \geq 0 .
\end{aligned}
$$

where $F_{\sigma_{M}}$ (resp. $f_{\sigma_{M}}$ ) denotes the distribution (resp. density) of the hitting time for the degradation level $M$ obtained using (2) and (3) respectively.

\section{Age-based maintenance models}

In this section, we are interested in the nature of the corrective maintenance actions (i.e. replacement or minimal repair) at failures due to shocks and in the optimal approach for the minimal repair decision (i.e. time-based or condition-based). When no restriction (or very few) are put on the decision rule structure, dynamic programming algorithms (Policy Iteration Algorithm for example) can be used to find optimal 
policies [40-42]. However, without reasonable restriction about the structure, it comes to be very difficult to formalize and to solve numerically the optimization problem and at last, from a practical point of view, the optimal strategy can be impossible to implement. Hence, an alternative reasonable approach is to fix some decision rule structures, to focus on the probabilistic model allowing their parameters optimization and to compare them with numerical studies. Even if in some cases, some results give indications about the structure of the optimal policy (for example in the framework of pure degradation models with monotone stationary markovian degradation phenomena [3, 43-47]), generally speaking, the selection of some specific decision rules is proposed after analyses of practical constraints and realistic considerations (see $[38,49]$ for example) to make sense. This selection can also be connected to the specific discussion objective of the research work to enhance some relevant conclusions in a specific context (value of preventive maintenance compared to corrective one, interest of condition based decision rules compared to periodic ones, value of the condition monitoring for the maintenance, ...).

In the present paper, we consider a complex case which is not a pure lifetime model, nor a pure degradation model. We consider a degradation model combined with a shock model. In the framework of systems subject to such competing failure modes, we do not think that the absolute optimality of one maintenance decision rule can be guaranteed theoretically. Hence we have a classic approach that is to fix some maintenance decision rules that are realistic from a practical point of view, that can be proved to be optimal in more simple cases, and that are relevant to evaluate the value of exact degradation level knowledge for decision making. Then, the major contribution of our paper is in the optimization of the parameters of these decision rules (with probabilistic calculation of the associated costs), and the participation to the recent discussions about the value of monitoring information that we address with the following question: is it necessary to inspect the system at failure time to know the origin of the failure (degradation or shock) and/or the system state at the failure (degradation level)? More precisely we analyze whether carrying out a minimal repair upon failure time due to a shock should be based:

- on the operational age of the system (age-based maintenance model with minimal repairs depending on the system age, $(\tau, T)$ maintenance policy)

- on the degradation of the system (age-based maintenance model with minimal repairs depending on the system degradation, $(A, T)$ maintenance policy).

Another age-based maintenance model without minimal repair is also introduced as a benchmark to assess the value of the minimal repairs in the maintenance decision-making. The analytic cost model of each maintenance policy is developed based on renewal considerations. Then numerical results in section 4 on a relevant study case give us a validation of the coherence of our decision rules and show the utility of our probabilistic quantification method for parameters optimization. 


\subsection{Assumptions and objective cost function}

\subsubsection{Assumptions}

Consider a repairable single-unit system described as in Section 2. We suppose that the system starts working at time $t=0$. The degradation of the system is hidden, and when the system fails, it can be self-announced but not reveal the failure type. It means that a system failure is immediately detected, but its degradation level and its failure type are only revealed through an inspection. These assumptions can be justified in practice because e.g. the system degradation and the failure type cannot be observed directly, due to the difficulty of placing sensors under harsh operating environments (high pressure, high temperature, high irradiation fields, etc.). However the system failure is usually easy to detect since the system stops working. The inspections are assumed to be instantaneous, perfect and non-destructive. After a failure, the system is immediately replaced or repaired by a maintenance action, hence it is never in a state of inactivity. Three maintenance operations of the system are available: the minimal repair with $\operatorname{cost} C_{m}$, the preventive replacement with $\operatorname{cost} C_{p}$, and the corrective replacement with cost $C_{c}$. A minimal repair is performed after a failure due to shocks and brings the system back to the same working conditions as it was just before the failure occurred. In our case, this means that it does not modify the degradation level of the system. A replacement can be either a physical replacement, an overhaul or a repair such that the system is as-goodas-new after the repair. Even though both the preventive and the corrective maintenance actions restore the system to the as-good-as-new state, they are not necessarily identical in practice. Since a corrective replacement (or renewal) is unplanned and has to be carried out on a more deteriorated system, it is more complex and expensive than a preventive replacement. The constraint of the maintenance operations costs is thus $C_{m}<C_{p}<C_{c}$. All the maintenance actions are assumed to take negligible time.

\subsubsection{Objective cost function}

To evaluate the performance of a maintenance policy, we focus on an asymptotic evaluation of a cost criterion which is the expected maintenance cost per unit time over an infinite time span. Assuming an as-good-asnew maintained system, an analytical formula of this long-run expected cost rate can be computed using the regenerative properties of the maintained system state [32]. We call a replacement cycle the time between two successive replacements of the system and we denote by $\sigma_{r}$ the time of a replacement cycle. Hence

$$
C_{\infty}=\lim _{t \rightarrow \infty} \frac{E[C(t)]}{t}=\frac{E\left[C\left(\sigma_{r}\right)\right]}{E\left[\sigma_{r}\right]}
$$

with probability 1 where $C(t)$ is the accumulated maintenance cost at time $t$. Thus, the long-run average cost per unit time is equal to the expected cost in a replacement cycle divided by the expected length of the replacement cycle for almost any realization of the process. 


\subsection{Age-based maintenance model with minimal repairs depending on the system age}

This section presents a modified age-based maintenance policy in which the decision of performing a minimal repair depends on the age $\tau$ of the system. Since the minimal repair decision is performed regardless of the actual condition of system, this policy is referred to as an age-based maintenance policy with "blind" minimal repairs and it is noted by $(\tau, T)$ policy. We also consider, in this policy, the system soft failure as the failure provoked by the shocks which arrive before age $\tau$, and hard failure as the failure provoked by the degradation or by the shocks which arrive after age $\tau$.

\subsection{1. $(\tau, T)$ maintenance policy}

Under the $(\tau, T)$ policy, if the system fails before the age $\tau<T$, an inspection with cost $C_{i f}$ is performed to check the type of failure. If the failure is due to degradation (i.e. hard failure), a corrective replacement with $\operatorname{cost} C_{c}$ is performed. If the failure is provoked by a shock (i.e. soft failure), a minimal repair with cost $C_{m}$ is performed. If the system fails after $\tau$, it is, by definition, a hard failure and a corrective replacement is performed regardless of the type of failure (i.e. without inspection). And if the system has not reached a hard failure state at age $T$, it is preventively replaced with cost $C_{p}$. Accordingly, $(\tau, T)$ policy reverts to the PAR policy (see subsection 3.4) when $\tau=0$. Practical conditions define the constraints of the maintenance costs as follows: $0<\tau, C_{i f}<C_{p}<C_{c}$ and $C_{m}<C_{p}<C_{c}$. The preventive replacement age $T$ and the maximum age to perform a minimal repair $\tau$ are the decision variables for this maintenance strategy. Figure 1 shows a sample behavior of the maintained system under this policy.

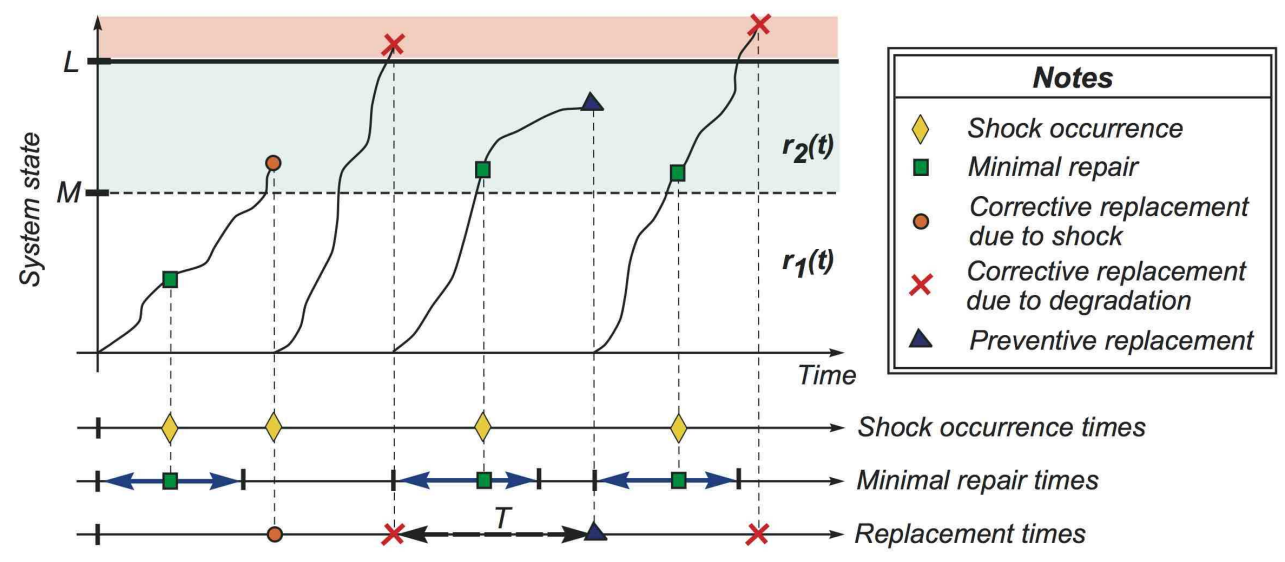

Figure 1: Schematic sample behavior of the maintained system under the $(\tau, T)$ policy

\subsubsection{Cost model formulation}

Let $\sigma_{f}$ be the time to a corrective maintenance, its distribution function $F_{f}$ at time $t$ is given by

$$
\bar{F}_{f}(\tau, t)=\bar{F}_{f, 1}(\tau, t) \cdot 1_{\{t \leq \tau\}}+\bar{F}_{f, 2}(\tau, t) \cdot 1_{\{t>\tau\}}
$$


where

$$
\bar{F}_{f, 1}(\tau, t)=P\left(\sigma_{L} \geq t\right)=\bar{F}_{\sigma_{L}}(t)
$$

and $\bar{F}_{\sigma_{L}}$ is given by equation (2). On the other hand,

$$
\begin{aligned}
\bar{F}_{f, 2}(\tau, t)= & P\left(\sigma_{L} \geq t, N_{s}(\tau, t]=0\right) \\
= & \frac{\bar{F}_{1}(t)}{\bar{F}_{1}(\tau)} \bar{F}_{\sigma_{M}}(t)+\frac{\bar{F}_{2}(t)}{\bar{F}_{1}(\tau)} \int_{\tau}^{t} \bar{F}_{\sigma_{L}-\sigma_{M}}(t-u) \frac{\bar{F}_{1}(u)}{\bar{F}_{2}(u)} f_{\sigma_{M}}(u) d u \\
& +\frac{\bar{F}_{2}(t)}{\bar{F}_{2}(\tau)} \int_{0}^{\tau} \bar{F}_{\sigma_{L}-\sigma_{M}}(t-u) f_{\sigma_{M}}(u) d u
\end{aligned}
$$

where $\bar{F}_{\sigma_{M}}, f_{\sigma_{M}}, \bar{F}_{\sigma_{L}-\sigma_{M}}$ and $\bar{F}_{i}, i=1,2$, are given by equations (2), (3), (4) and (6) respectively.

Hence the probability of a preventive maintenance in a replacement cycle is given by

$$
P_{p}^{\tau T}(\tau, T)=\bar{F}_{f}(\tau, T)
$$

and the probability of a corrective maintenance in a replacement cycle is therefore given by

$$
P_{c}^{\tau T}(\tau, T)=1-P_{p}^{\tau T}(\tau, T)=F_{f}(\tau, T)
$$

Inspections are performed when the system fails before the age $\tau$. Let $E\left[N_{i}^{\tau T}(t)\right]$ be the expected number of inspections effected until the time $t \leq \tau$, then it can be computed by

$$
E\left[N_{i}^{\tau T}(t)\right]=\int_{0}^{t} r_{1}(u) \bar{F}_{\sigma_{M}}(u) d u+\int_{0}^{t} f_{\sigma_{M}}(u)\left(\int_{u}^{t} r_{2}(v) \bar{F}_{\sigma_{L}-\sigma_{M}}(v-u) d v\right) d u+F_{\sigma_{L}}(t) .
$$

Therefore, the expected number of inspections in a replacement cycle $E\left[N_{i}^{\tau T}(\tau, T)\right]$ is given by

$$
E\left[N_{i}^{\tau T}(\tau, T)\right]=E\left[N_{i}^{\tau T}(T)\right] \cdot 1_{\{T \leq \tau\}}+E\left[N_{i}^{\tau T}(\tau)\right] \cdot 1_{\{T>\tau\}} .
$$

The number of minimal repairs is equal to the number of inspections minus the number of failures due to degradation before the age $\tau$. Let $E\left[N_{m r}^{\tau T}(t)\right]$ be the expected number of minimal repairs effected until the time $t \leq \tau$, then it can be computed by

$$
E\left[N_{m r}^{\tau T}(t)\right]=E\left[N_{i}^{\tau T}(t)\right]-F_{\sigma_{L}}(t)
$$

where $E\left[N_{i}^{\tau T}(t)\right]$ is given by (12). Therefore, the expected number of minimal repairs in a replacement cycle $E\left[N_{m r}^{\tau T}(\tau, T)\right]$ is given by

$$
E\left[N_{m r}^{\tau T}(\tau, T)\right]=E\left[N_{m r}^{\tau T}(T)\right] \cdot 1_{\{T \leq \tau\}}+E\left[N_{m r}^{\tau T}(\tau)\right] \cdot 1_{\{T>\tau\}} .
$$

From equation (8), the expected length of a replacement cycle is given by

$$
E\left[\sigma_{r}^{\tau T}(\tau, T)\right]=E\left[\sigma_{r, 1}^{\tau T}(\tau, T)\right] \cdot 1_{\{T \leq \tau\}}+E\left[\sigma_{r, 2}^{\tau T}(\tau, T)\right] \cdot 1_{\{T>\tau\}}
$$


where

$$
E\left[\sigma_{r, 1}^{\tau T}(\tau, T)\right]=\int_{0}^{T} \bar{F}_{\sigma_{L}}(t) d t
$$

and

$$
E\left[\sigma_{r, 2}^{\tau T}(\tau, T)\right]=\int_{0}^{\tau} \bar{F}_{\sigma_{L}}(t) d t+\int_{\tau}^{T} \bar{F}_{f, 2}(\tau, t) d t
$$

where $\bar{F}_{f, 2}$ is given by (9). Taking into account equations (7), (10), (11), (13), (14) and (15), the expression of the expected cost rate for this maintenance model $C_{\infty}^{\tau T}(\tau, T)$ is given by

$$
C_{\infty}^{\tau T}(\tau, T)=\frac{C_{c} P_{c}^{\tau T}(\tau, T)+C_{p} P_{p}^{\tau T}(\tau, T)+C_{i f} E\left[N_{i}^{\tau T}(\tau, T)\right]+C_{m} E\left[N_{m r}^{\tau T}(\tau, T)\right]}{E\left[\sigma_{r}^{\tau T}(\tau, T)\right]} .
$$

The optimization problem for this maintenance strategy is reduced to find the values $\tau$ and $T$ that minimize $C_{\infty}^{\tau T}(\tau, T)$ given by $(16)$, that is

$$
C_{\infty}^{\tau T}\left(\tau_{\text {opt }}, T_{\text {opt }}\right)=\inf \left\{C_{\infty}^{\tau T}(\tau, T), \tau>0, T>0\right\}
$$

\subsubsection{Numerical example}

To have a numerical illustration for the expected cost rate and the optimal maintenance strategy of the $(\tau, T)$ policy, we use the following data set: $\alpha=\beta=1, L=30, M=20, r_{1}(t)=\lambda_{1}=0.05, r_{2}(t)=\lambda_{2}=0.5$, $C_{i f}=20, C_{m}=40, C_{p}=50$ and $C_{c}=100$. This data set represents a case of intermediate variance in degradation increment $\left(m=\frac{\alpha}{\beta}=1, \sigma^{2}=\frac{\alpha}{\beta^{2}}=1\right)$ and high intensity of failures due to shocks. This numerical example is illustrated in Figure 2. Figure 2 shows the expected cost rate of the $(\tau, T)$ policy as

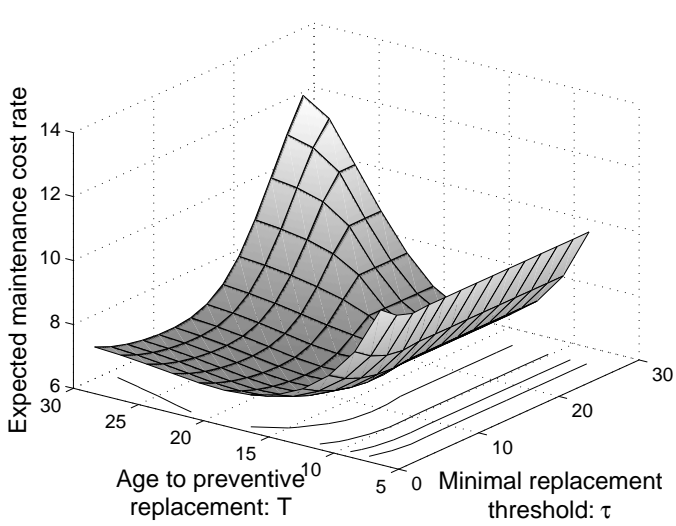

(a) Mesh of expected maintenance cost

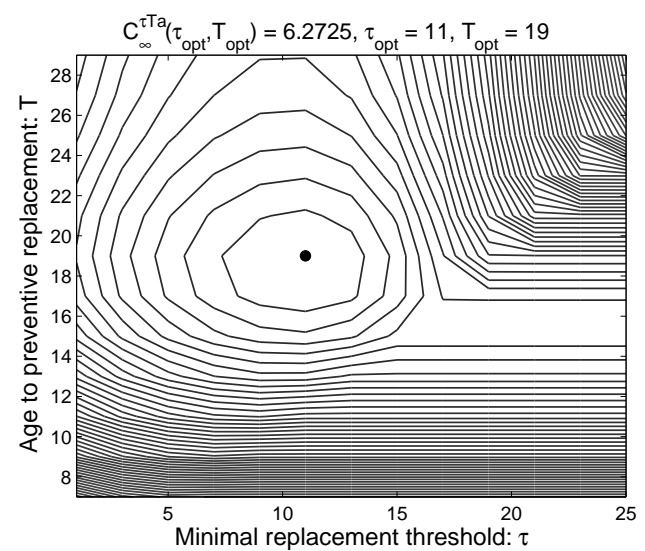

(b) Iso-level cost curves of expected maintenance cost

Figure 2: Optimization of the age-based maintenance policy with minimal repairs depending on the system age - $(\tau, T)$ policy

a function of the preventive replacement age $T$ and the minimal repair threshold $\tau$. It can be seen that we 
can obtain an optimal solution under the $(\tau, T)$ policy, and in this case, the optimal values of the decision variables (obtained by a classical optimization scheme) are $T_{o p t}=19$ and $\tau_{o p t}=11$ with an optimal cost rate given by $C_{\infty}^{\tau T}\left(\tau_{\text {opt }}, T_{\text {opt }}\right)=6.2725$ monetary units.

\subsection{Age-based maintenance model with minimal repairs depending on the system degradation}

The $(\tau, T)$ policy developed in Section 3.2 seems to be an effective age-based maintenance policy with minimal repairs. However, minimal repairs are based on the age of the system at the time of failure which can be less effective than the decisions based on the condition of the system in some situations [33, 34]. Therefore, this section aims to develop a more complex age-based maintenance policy in the sense that the minimal repair decision is performed depending on the actual condition of system, and this policy is called $(A, T)$ policy. We define, in this policy, a system soft failure as the failure provoked by the occurrence of a shock when the degradation level does not exceed the degradation value $A$, and as a hard failure as the failure provoked by the degradation, or by a shock which has occurred when the degradation level exceeds the value $A$.

\subsection{1. $(A, T)$ maintenance policy}

Under this maintenance strategy, if the system is not in a hard failure state at the age $T$, a preventive replacement is performed with a cost $C_{p}$. If the system stopped working before the age $T$, an inspection with $\operatorname{cost} C_{i d}$ is performed to check the type of failure and the degradation level of system. If the inspection detects the system is in a hard failure state, a corrective replacement is performed with a $\operatorname{cost} C_{c}$. But if the system is just in a soft failure state, a minimal repair is performed with an additional $\operatorname{cost} C_{m}$. Practical conditions define the constraint of the maintenance costs as follows: $0<A<L, C_{i d}<C_{p}<C_{c}$ and $C_{m}<C_{p}<C_{c}$. The preventive replacement age period $T$ and the maximum degradation level to perform a minimal repair $A$ are the decision variables for this maintenance strategy. Figure 3 shows a sample behavior of the maintained system under this policy.

Comparing the $(A, T)$ strategy with the $(\tau, T)$ strategy, inspection costs verify the inequality $C_{i d} \geq C_{i f}$. It means that an inspection under the $(A, T)$ policy is more expensive than an inspection under the $(\tau, T)$ policy. The reason for this is that in the $(A, T)$ policy, the inspection determines not only the type of failure (due to degradation or due to shock), but also the degradation level of the system.

\subsubsection{Cost model formulation}

Let $\sigma_{f s}$ be the time to a system replacement due to shocks,

$$
\sigma_{f s}=\inf \left\{t \geq 0, N_{s}(t)-N_{s}\left(0, \sigma_{A}\right)=1, X(t)>A\right\},
$$

where $\sigma_{A}$ denotes the first hitting time to the level $A$. 


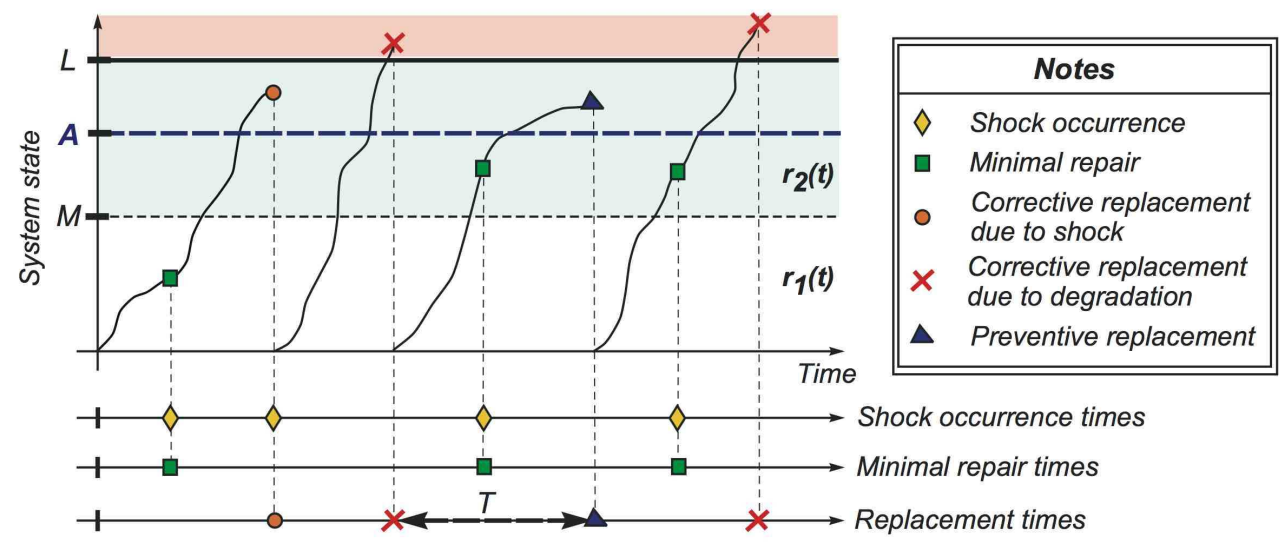

Figure 3: Schematic sample behavior of the maintained system under the $(A, T)$ policy

Let $\sigma_{f}$ be the time to the system replacement due to a hard failure, then

$$
\sigma_{f}=\min \left(\sigma_{L}, \sigma_{f s}\right)
$$

where $\sigma_{L}$ and $\sigma_{f s}$ are given by (1) and (17) respectively. The survival function of the time to the system replacement $\sigma_{f}$ is given by

$$
\bar{F}_{f}(t)=P\left(\sigma_{f}>t\right)=E\left[1_{\left\{\sigma_{A}>t\right\}}+1_{\left\{\sigma_{L}>t>\sigma_{A}\right\}} \exp \left(-\int_{\sigma_{A}}^{t} r(X(s)) d s\right)\right], \quad t \geq 0 .
$$

The survival function of the time to the system replacement $\sigma_{f}$ can also be expressed by

$$
\bar{F}_{f}(A, t)=\bar{F}_{f, 1}(A, t) \cdot 1_{\{A \leq M\}}+\bar{F}_{f, 2}(A, t) \cdot 1_{\{A>M\}},
$$

where

$$
\begin{aligned}
\bar{F}_{f, 1}(A, t)= & \bar{F}_{\sigma_{A}}(t)+\bar{F}_{1}(t) \int_{0}^{t} \bar{F}_{\sigma_{M}-\sigma_{A}}(t-u) \frac{1}{\bar{F}_{1}(u)} f_{\sigma_{A}}(u) d u \\
& +\bar{F}_{2}(t) \int_{0}^{t} \int_{u}^{t} \bar{F}_{\sigma_{L}-\sigma_{M}}(t-v) \frac{\bar{F}_{1}(v)}{\bar{F}_{1}(u) \bar{F}_{2}(v)} f_{\sigma_{M}-\sigma_{A}}(v-u) f_{\sigma_{A}}(u) d v d u
\end{aligned}
$$

and

$$
\bar{F}_{f, 2}(A, t)=\bar{F}_{\sigma_{A}}(t)+\bar{F}_{2}(t) \int_{0}^{t} \bar{F}_{\sigma_{L}-\sigma_{A}}(t-u) \frac{1}{\bar{F}_{2}(u)} f_{\sigma_{A}}(u) d u .
$$

Hence the probability of a preventive maintenance in a replacement cycle is given by

$$
P_{p}^{A T}(A, T)=\bar{F}_{f}(A, T)
$$

and the probability of a corrective maintenance in a replacement cycle is given by

$$
P_{c}^{A T}(A, T)=1-P_{p}^{A T}(A, T)=F_{f}(A, T) .
$$


The expected length of the renewal cycle is obtained as

$$
E\left[\sigma_{r}^{A T}(A, T)\right]=\int_{0}^{T} \bar{F}_{f}(A, t) d t .
$$

Considering the assumptions of the maintenance strategy, if no replacement is performed, the expected number of minimal repairs at time $t$ is given by

$$
E\left[N_{m r}^{A T}(A, t)\right]=E\left[N_{s}(t) \cdot 1_{\left\{\sigma_{A}>t\right\}}+N_{s}\left(\sigma_{A}\right) \cdot 1_{\left\{\sigma_{A} \leq t\right\}}\right], \quad t \geq 0 .
$$

Therefore, the expected number of minimal repairs in a replacement cycle $E\left[N_{m r}^{A T}(A, T)\right]$ is given by

$$
E\left[N_{m r}^{A T}(A, T)\right]=E\left[N_{m r, 1}^{A T}(A, T)\right] \cdot 1_{\{A \leq M\}}+E\left[N_{m r, 2}^{A T}(A, T)\right] \cdot 1_{\{A>M\}},
$$

where

$$
E\left[N_{m r, 1}^{A T}(A, T)\right]=\int_{0}^{T} r_{1}(u) \bar{F}_{\sigma_{A}}(u) d u,
$$

and

$$
E\left[N_{m r, 2}^{A T}(A, T)\right]=\int_{0}^{T} r_{1}(u) \bar{F}_{\sigma_{M}}(u) d u+\int_{0}^{T} \int_{u}^{T} r_{2}(z) \bar{F}_{\sigma_{A}-\sigma_{M}}(z-u) f_{\sigma_{M}}(u) d z d u .
$$

Taking into account equations (7), (18), (19), (20) and (21), the expected cost rate for this maintenance model $C_{\infty}^{A T}(A, T)$ is given by

$$
C_{\infty}^{A T}(A, T)=\frac{\left(C_{c}+C_{i d}\right) P_{c}^{A T}(A, T)+C_{p} P_{p}^{A T}(A, T)+\left(C_{m}+C_{i d}\right) E\left[N_{m r}^{A T}(A, T)\right]}{E\left[\sigma_{r}^{A T}(A, T)\right]}, \quad 0<A<L, T>0 .
$$

The optimization problem for this maintenance strategy is reduced to find the values $A$ and $T$ that minimize $C_{\infty}^{A T}(A, T)$ given by $(22)$, that is

$$
C_{\infty}^{A T}\left(A_{\text {opt }}, T_{\text {opt }}\right)=\inf \left\{C_{\infty}^{A T}(A, T), 0<A<L, T>0\right\} .
$$

\subsubsection{Numerical example}

For a numerical illustration of the $(A, T)$ policy, we use in this section the same data set given in the numerical example of Section 3.2. To obtain a preliminary estimation of the profit gained by the $(A, T)$ policy compared to the $(\tau, T)$ policy, we set the inspection cost $C_{i d}$ equal to $C_{i f}$. Figure 4 shows the expected maintenance cost rate as a function of decision variables $A$ and $T$. The optimal solution is obtained at $T_{o p t}=17$ and $A_{\text {opt }}=17$ with an optimal expected cost rate of $C_{\infty}^{A T}\left(A_{\text {opt }}, T_{\text {opt }}\right)=6.4621$ monetary units.

We notice that, though the $(A, T)$ policy is more complex than the $(\tau, T)$ policy from the point of view of the maintenance structure, its optimal maintenance cost rate can be higher $\left(C_{\infty}^{A T}\left(A_{o p t}, T_{\text {opt }}\right)=6.4621\right.$ v.s. $\left.C_{\infty}^{\tau T}\left(\tau_{o p t}, T_{o p t}\right)=6.2725\right)$. But there are some situations where the $(A, T)$ policy can save costs when compared to the $(\tau, T)$ policy, because it allows for a timely minimal repair thanks to the decision based on the actual condition of the system. So a detailed analysis on the optimal expected maintenance cost of these maintenance policies under the different possible situations of system characteristics and maintenance cost is essential to select an adequate strategy of maintenance. This will be elucidated in subsection 4.2.3. 


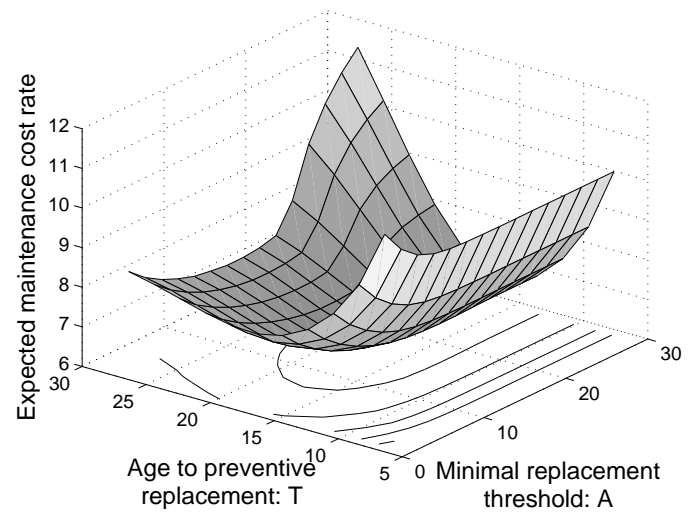

(a) Mesh of expected maintenance cost

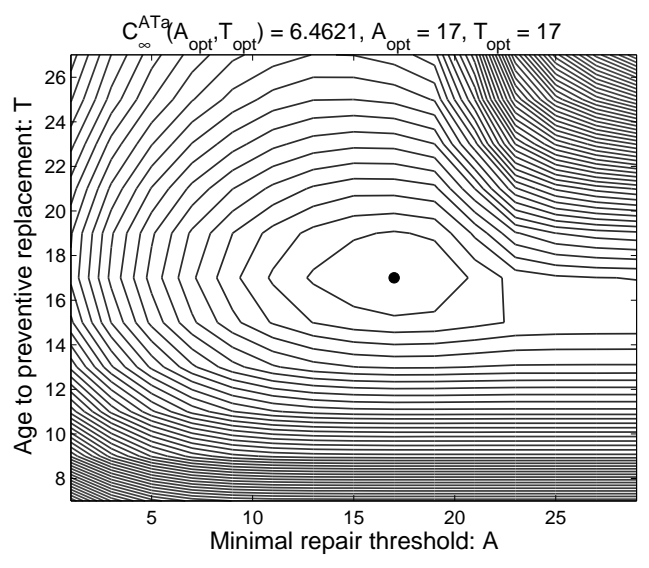

(b) Iso-level cost curves of expected maintenance cost

Figure 4: Optimization of the age-based maintenance policy with minimal repairs depending on the system degradation - $(A, T)$ policy

\subsection{Pure age-based replacement model and synthesis of maintenance policies}

This section introduces a classical maintenance model as benchmark to assess the advantages of the two proposed maintenance policies (i.e. $(A, T)$ policy and $(\tau, T)$ policy), and the effect of the minimal repair in the maintenance decision. Then we summarize the considered maintenance model through a comparison table. The summary table shows the differences in the approach of the minimal repair decision of the maintenance policies.

\subsubsection{Pure age-based replacement model}

This pure age-based replacement policy (PAR policy) is representative of the class of maintenance strategies without minimal repairs. In the present paper, this strategy is used as a benchmark to assess the performance of the age-based maintenance strategies with minimal repairs.

1. Pure age-based replacement policy [35]. Under this maintenance policy, the system is correctively replaced upon failure (i.e. when the degradation of the system exceeds the value $L$ or when a shock occurs) or preventively replaced at a specified operational age $T$, whichever occurs first. A preventive (resp. corrective) replacement is performed with a cost $C_{p}$ (resp. $C_{c}>C_{p}$ ). The preventive replacement age period $T$ is the only decision variable for this policy.

When we compare $(\tau, T)$ and $(A, T)$ policies with a PAR policy, additional inspection costs are incurred to find out the failure type and/or the degradation level at the failure time but we can decide to keep a not-too-worn system in use thanks to any minimal repairs undertaken. 
2. Cost model formulation. Applying equation (7), the expected cost rate for this maintenance model is given by

$$
C_{\infty}^{p}(T)=\frac{C_{c} F_{f}(T)+C_{p} \bar{F}_{f}(T)}{\int_{0}^{T} \bar{F}_{f}(t) d t}, \quad T>0,
$$

where $\bar{F}_{f}(t)$ denotes the survival distribution of the time of failure for the system,

$$
\begin{aligned}
\bar{F}_{f}(t) & =P\left[\sigma_{L} \geq t, N_{s}(t)=0\right] \\
& =\bar{F}_{1}(t) \cdot \bar{F}_{\sigma_{M}}(t)+\bar{F}_{2}(t) \int_{0}^{t} \bar{F}_{\sigma_{L}-\sigma_{M}}(t-u) \frac{\bar{F}_{1}(u)}{\bar{F}_{2}(u)} f_{\sigma_{M}}(u) d u, \quad t \geq 0
\end{aligned}
$$

where $\bar{F}_{\sigma_{M}}, f_{\sigma_{M}}, \bar{F}_{\sigma_{L}-\sigma_{M}}$ and $\bar{F}_{i}, i=1,2$ are given from equations (2), (3), (4) and (6) respectively. The optimization problem for this maintenance strategy is to find a value of $T$ that minimizes $C_{\infty}^{p}(T)$ given by equation (23), that is

$$
C_{\infty}^{p}\left(T_{o p t}\right)=\inf \left\{C_{\infty}^{p}(T), \quad T>0\right\}
$$

\subsubsection{Synthesis of maintenance strategies developed in this paper}

The summarized characteristics of the considered maintenance models are listed in Table 1. Table 1 shows and compares the differences in the nature of the maintenance decision from the viewpoint of the maintenance structure of each model.

\begin{tabular}{|c|c|c|c|c|c|}
\hline Policy & $\mathbf{P R}^{a}$ decision & $\mathbf{C R}^{a}$ decision & $\mathbf{M R}^{a}$ decision & Inspection for & Optimization \\
\hline \hline$(A, T)$ policy & $\begin{array}{c}\text { at } T \text { (no hard } \\
\text { failure before) }\end{array}$ & at hard failure & $\begin{array}{c}\text { condition-based }(A) \\
\text { at soft failure }\end{array}$ & $\begin{array}{c}\text { degradation \& } \\
\text { failure types }\left(C_{i d}\right)\end{array}$ & $A, T$ \\
\hline$(\tau, T)$ policy & $\begin{array}{c}\text { at } T \text { (no hard } \\
\text { failure before) }\end{array}$ & at hard failure & $\begin{array}{c}\text { age-based }(\tau) \\
\text { at soft failure }\end{array}$ & $\begin{array}{c}\text { failure types } \\
\left(C_{i f}<C_{i d}\right)\end{array}$ & $\tau, T$ \\
\hline PAR policy & $\begin{array}{c}\text { at } T \text { (no } \\
\text { failure before) }\end{array}$ & at failure & $\varnothing$ & $\varnothing$ & $T$ \\
\hline
\end{tabular}

${ }^{a} \mathrm{PR}$ : preventive replacement - CR: corrective replacement - MR: minimal repair

Table 1: Summary of the age-based maintenance policies for the considered degradation/failure model

The fundamental difference between the age-based maintenance policies with and without minimal repairs is the maintenance decision at a failure due to shocks. For the PAR policy, it is always a corrective replacement, while for the strategies with minimal repairs, this can be either a corrective replacement or a minimal repair depending on the observed situations. Of course, for the maintenance strategies with minimal repairs, an additional inspection cost is incurred in order to detect the type of failure or, even, the degradation level at the failure time. An interesting problem is which maintenance policy should be applied according to given system characteristics and maintenance costs. The next section aims to answer this question. 


\section{Analysis and discussion of the numerical results}

To obtain a thorough analysis of the effectiveness of each maintenance policy, as well as the value of the minimal repair in the maintenance decision, we carry out, in this section, numerical comparisons on the expected cost rate among the considered maintenance models under different characteristics of the system (i.e. variance of the degradation process, degradation speed, shock failure rate, and maintenance costs). The numerical results will be the indicators to justify or not the choice to implement minimal repairs in the maintenance strategies and to invest in condition monitoring.

\subsection{Relative gain on the optimal maintenance cost rate}

In order to compare the performance of the different maintenance strategies, criteria on the relative gain $(\mathrm{RG})$ in the optimal maintenance cost rate are introduced. The RG of the policy A compared to the policy $\mathrm{B}$ is computed as follows

$$
R G_{A / B}=\frac{C_{\infty}^{B, o p t}-C_{\infty}^{A, o p t}}{C_{\infty}^{B, o p t}}
$$

where $C_{\infty}^{A, o p t}$ and $C_{\infty}^{B, o p t}$ are the optimal maintenance cost rates of the policies $\mathrm{A}$ and $\mathrm{B}$ respectively. If $R G_{A / B}>0$, policy $\mathrm{A}$ is more profitable than policy $\mathrm{B}$ in terms of the maintenance cost. If $R G_{A / B}<0$, policy $\mathrm{A}$ is less profitable than policy $\mathrm{B}$ in terms of the maintenance cost. And $R G_{A / B}=0$ means that the policy A and B have the same optimal maintenance cost.

\subsection{Numerical results and discussions}

We are interested in the effect of minimal repair cost and inspection cost on the performance of each maintenance policy considered, so we vary these costs and fix the preventive replacement cost $C_{p}=50$ and the corrective replacement $\operatorname{cost} C_{c}=100$. The degradation level that changes the shock failure rate $M$ and the degradation failure threshold $L$ are chosen by $M=20$ and $L=30$.

\subsection{1. $(\tau, T)$ policy vs. PAR policy}

In order to illustrate the use of our model to assess the advantage of time-based minimal repairs as possible corrective maintenance actions, we vary the inspection cost and the minimal repair cost together, and observe the RG of the $(\tau, T)$ policy compared to the PAR policy under different characteristics of the maintained system. We are interested in the impact of the degradation variance, the degradation speed, and the failure rate due to shocks on the maintenance cost of each maintenance policy, so four different cases of system behavior are considered:

1. Case 1. Low variance in degradation increment, low degradation speed and high intensity to shock failures: $\alpha=\beta=1\left(\sigma^{2}=1, m=1\right), r_{1}(t)=\lambda_{1}=0.1, r_{2}(t)=\lambda_{2}=0.5$.

2. Case 2. High variance in degradation increment, low degradation speed and high intensity to shock failures: $\alpha=\beta=0.1\left(\sigma^{2}=10, m=1\right), r_{1}(t)=\lambda_{1}=0.1, r_{2}(t)=\lambda_{2}=0.5$. 
3. Case 3. High variance in degradation increment, high degradation speed and high intensity to shock failures: $\alpha=0.4, \beta=0.2\left(\sigma^{2}=10, m=2\right), r_{1}(t)=\lambda_{1}=0.1, r_{2}(t)=\lambda_{2}=0.5$.

4. Case 4. High variance in degradation increment, high degradation speed and low intensity to shock failures: $\alpha=0.4, \beta=0.2\left(\sigma^{2}=10, m=2\right), r_{1}(t)=\lambda_{1}=0.01, r_{2}(t)=\lambda_{2}=0.05$.

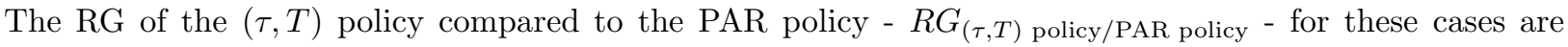
represented in Figure 5.

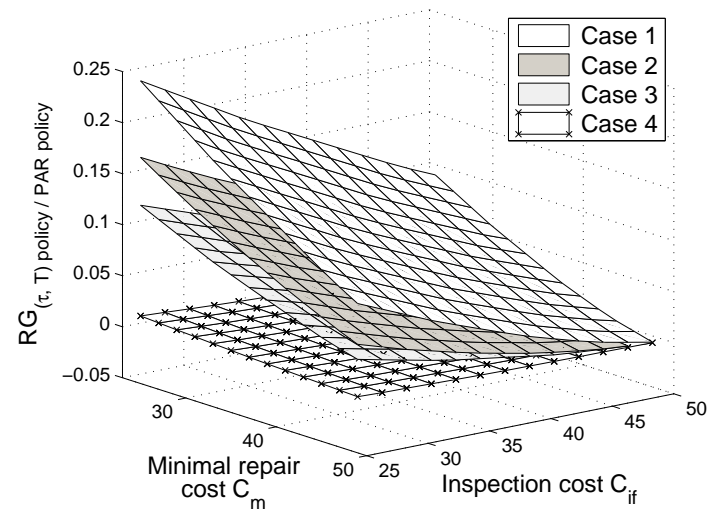

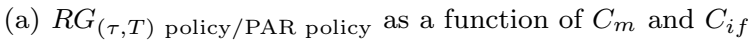

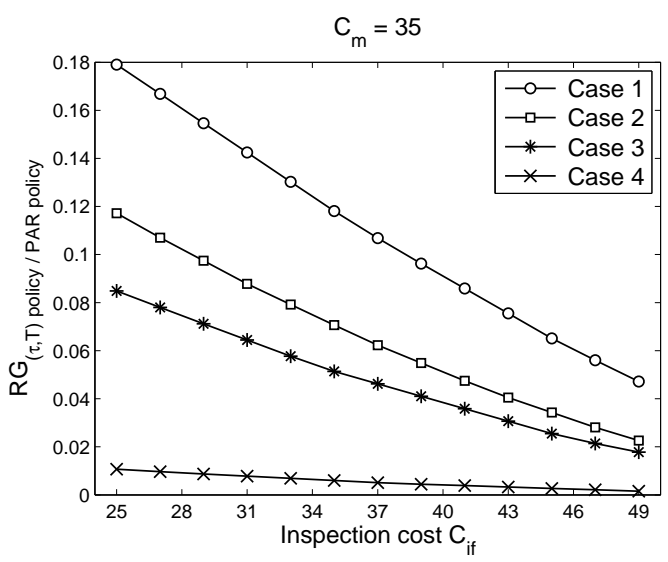

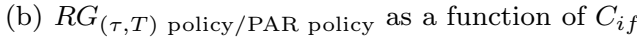

Figure 5: Relative gain on the optimal maintenance cost rate of the $(\tau, T)$ policy compared to the PAR policy

Obviously, the RG of the $(\tau, T)$ policy compared to the PAR policy decreases with the increase of the inspection $\operatorname{cost} C_{i f}$ and/or the minimal repair cost $C_{m}$. Figure 5 shows that the higher the intensity to shock failures and the lower the degradation speed and variance, the more the $(\tau, T)$ policy is profitable against the PAR policy. It can be explained by the fact that minimal repairs are performed more frequently with high intensity to shock failure and low speed of degradation process; moreover since the sum of inspection $\operatorname{cost} C_{i f}$ and minimal repair cost $C_{m}$ is usually so much smaller than a corrective replacement cost $C_{c}$, so in this case, the proposed $(\tau, T)$ policy can lead to more profit (e.g. case 2 vs. case 3 , and case 3 vs. case 4 ). Furthermore, the minimal repair in the $(\tau, T)$ policy is based on the age of the system which is more suitable for a smoother behavior of the degradation process, hence the $(\tau, T)$ policy is more profitable in terms of cost when the variance of the degradation process is low (e.g. case 1 vs. case 2). Since the PAR policy is a special case of $(\tau, T)$ policy, the latter can always lead to more savings in maintenance cost compared with the former for any given system characteristics and maintenance $\operatorname{costs}\left(R G_{(\tau, T) \text { policy/PAR policy }} \geq 0\right)$. Hence it is always preferable to apply a maintenance policy with time-based minimal repairs instead of a classical pure-age based replacement policy for a repairable system. 


\subsection{2. $(A, T)$ policy vs. PAR policy}

To show the interest of resorting to minimal repairs depending on the degradation level as corrective maintenance actions, we study the evolution of RG of the $(A, T)$ policy compared to the PAR policy under different characteristics of the maintained system by varying both the inspection cost and the minimal repair cost. We are interested in the influence of the degradation variance, the degradation speed and the shock failure rate on the cost of the $(A, T)$ policy against the PAR policy, so we consider four cases of system behaviors:

1. Case 1. High variance in degradation increment, low degradation speed and high intensity to shock failures: $\alpha=\beta=0.1\left(\sigma^{2}=10, m=1\right), r_{1}(t)=\lambda_{1}=0.1, r_{2}(t)=\lambda_{2}=0.5$.

2. Case 2. Low variance in degradation increment, low degradation speed and high intensity to shock failures: $\alpha=\beta=1\left(\sigma^{2}=1, m=1\right), r_{1}(t)=\lambda_{1}=0.1, r_{2}(t)=\lambda_{2}=0.5$.

3. Case 3. Low variance in degradation increment, low degradation speed and low intensity to shock failures: $\alpha=\beta=1\left(\sigma^{2}=1, m=1\right), r_{1}(t)=\lambda_{1}=0.0375, r_{2}(t)=\lambda_{2}=0.1875$.

4. Case 4. Low variance in degradation increment, high degradation speed and low intensity to shock failures: $\alpha=4, \beta=2\left(\sigma^{2}=1, m=2\right), r_{1}(t)=\lambda_{1}=0.0375, r_{2}(t)=\lambda_{2}=0.1875$.

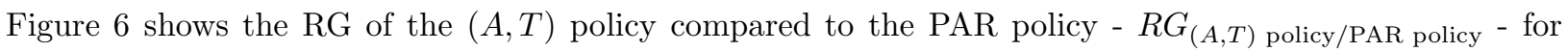
these cases.

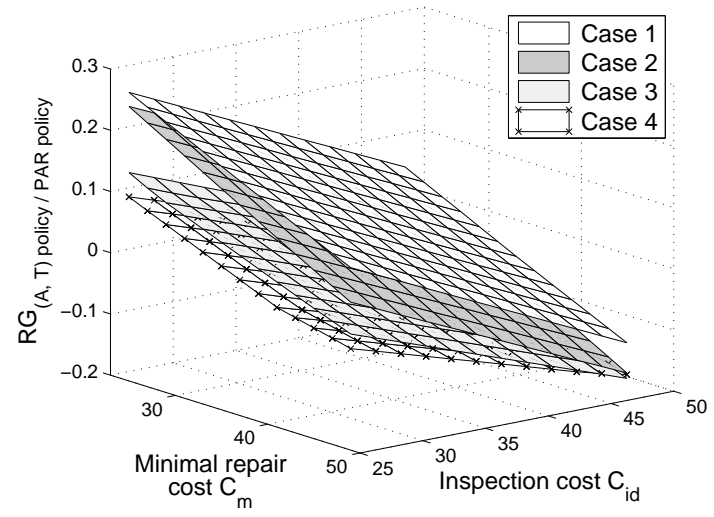

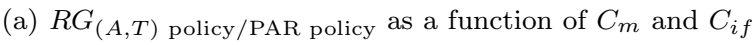

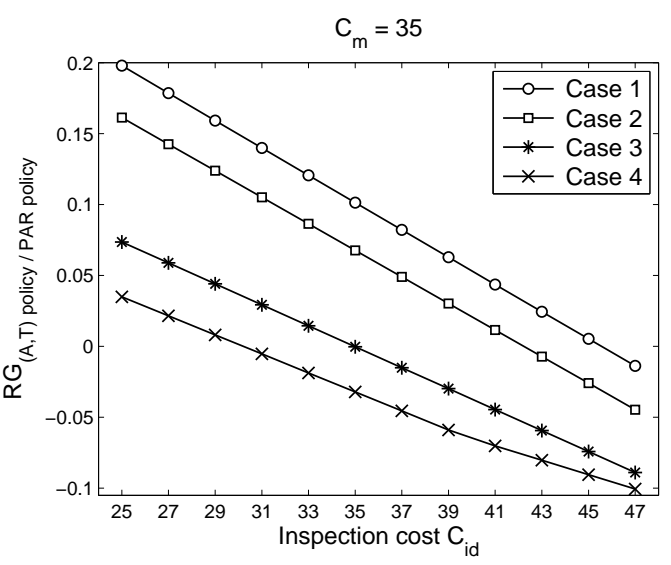

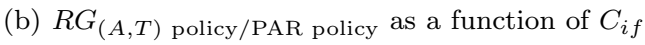

Figure 6: Relative gain on the optimal maintenance cost rate of the $(A, T)$ policy compared to the PAR policy

Not surprisingly, the levels of $R G_{(A, T)}$ policy/PAR policy always decrease according to the increase in the inspection cost $C_{i f}$ and/or minimal repair cost $C_{m}$. We can see in Figure 6 that the $(A, T)$ policy may lead to a significant saving in the maintenance cost when the inspections and/or minimal repairs are not too expensive. For the same reason as in Section 4.2.1, the $(A, T)$ policy becomes more profitable when the 
intensity to shock failure becomes higher (e.g. case 2 vs. case 3 ) and the degradation speed is lower (e.g. case 3 vs. case 4$)$. The benefit of the $(A, T)$ policy compared to the PAR policy is more evident in the case of high variance in the degradation increment (e.g. case 1 vs. case 2), as in this case, the degradation level gives a lot of information on the actual condition of the system and thus the minimal repair decision based on the degradation level can give a timely intervention, leading to more savings in maintenance costs. However, the $(A, T)$ policy is not always more profitable than the PAR policy (e.g. in the case of high minimal repair cost and inspection cost, and/or in the case of low failure rate of shock and low variance of degradation). Therefore, even if it may be interesting to perform a condition-based minimal repair, one needs to be cautious about the system characteristics and maintenance costs when making a decision.

\subsection{3. $(A, T)$ policy vs. $(\tau, T)$ policy}

The numerical results in the subsections above show that the introduction of minimal repair can lead to a significant saving in some maintenance strategies for a repairable system. However between the $(\tau, T)$ policy and the $(A, T)$ policy, which one can yield more savings, and under which conditions which one of them is the more appropriate? To answer this question, we vary the inspection cost in $(\tau, T)$ policy $C_{i f}$ and the difference between the inspection costs of both policies $\Delta C_{i}=C_{i d}-C_{i f}$ together, and investigate the evolution of the corresponding $\mathrm{RG}$ of the $(A, T)$ policy against the $(\tau, T)$ policy - $R G_{(A, T) \text { policy } /(\tau, T) \text { policy }}$ - under different characteristics of the maintained system and minimal repair costs.

To study the sensitivity to the dynamic behavior of system, we fix the minimal repair cost $C_{m}=30$ and consider the following case studies:

1. Case 1. High variance in the degradation increment and high intensity to shock failures: $\alpha=\beta=0.1$ $\left(\sigma^{2}=10\right), r_{1}(t)=\lambda_{1}=0.1, r_{2}(t)=\lambda_{2}=0.5$.

2. Case 2. Low variance in the degradation increment and high intensity to shock failures: $\alpha=\beta=1$ $\left(\sigma^{2}=1\right), r_{1}(t)=\lambda_{1}=0.1, r_{2}(t)=\lambda_{2}=0.5$.

3. Case 3. High variance in the degradation increment and low intensity to shock failures: $\alpha=\beta=0.1$ $\left(\sigma^{2}=10\right), r_{1}(t)=\lambda_{1}=0.025, r_{2}(t)=\lambda_{2}=0.125$.

To study the sensitivity to the minimal repair costs, we fix the system characteristics by the data set: $\alpha=\beta=0.1, r_{1}(t)=\lambda_{1}=0.1, r_{2}(t)=\lambda_{2}=0.5$ and we consider different values for the minimal repair cost $C_{m}=5,25$, and 45 respectively.

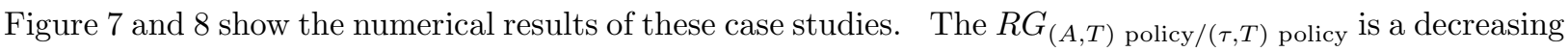
function of $C_{i d}-C_{i f}$ and a concave function of $C_{i f}$. When $\Delta C_{i}$ is small (i.e. the inspection in $(A, T)$ policy is not so expensive compared to the inspection in $(\tau, T)$ policy), $(A, T)$ policy seems to have an advantage over the $(\tau, T)$ policy. Nevertheless, it is hard to give a general conclusion about the benefit of each maintenance policy. The reason being that compared to the $(\tau, T)$ policy, the cost saving of $(A, T)$ policy depends largely 


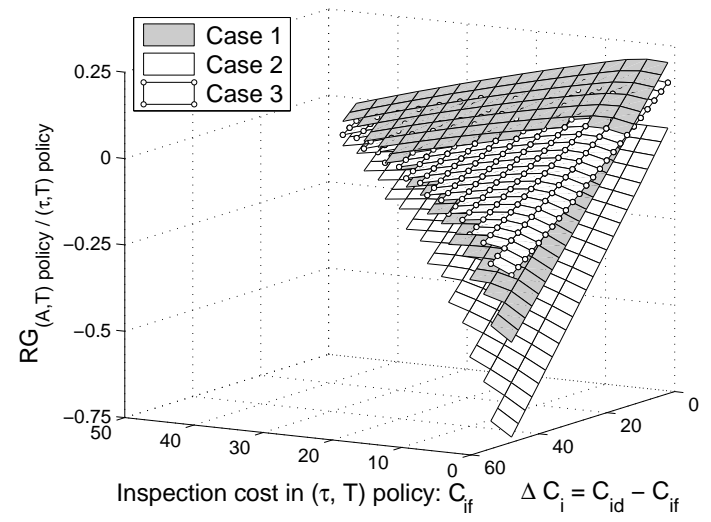

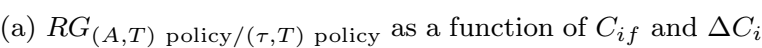
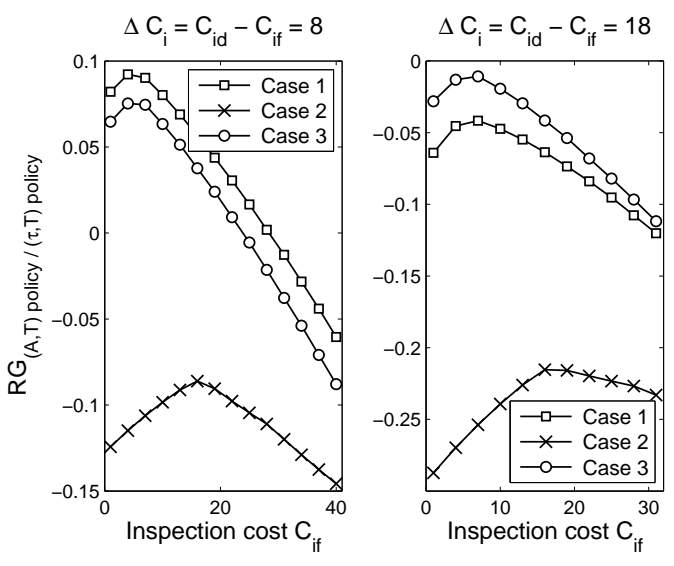

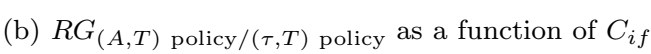

Figure 7: Relative gain on the optimal maintenance cost rate of the $(A, T)$ policy compared to the $(\tau, T)$ policy for different system behaviors

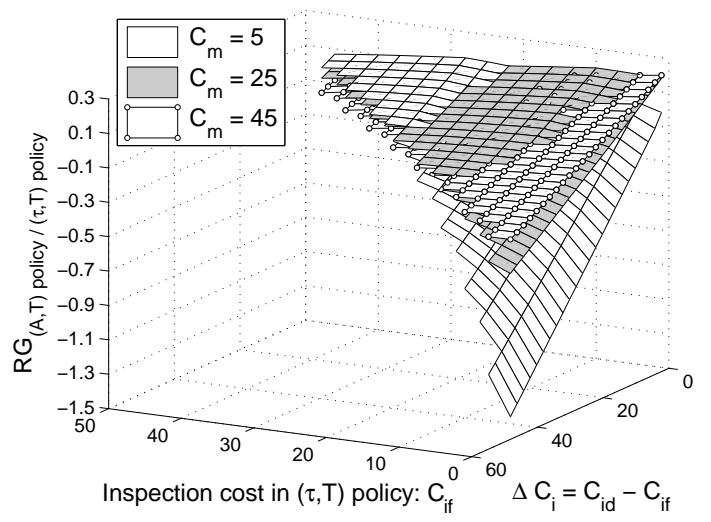

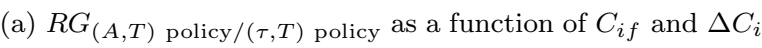
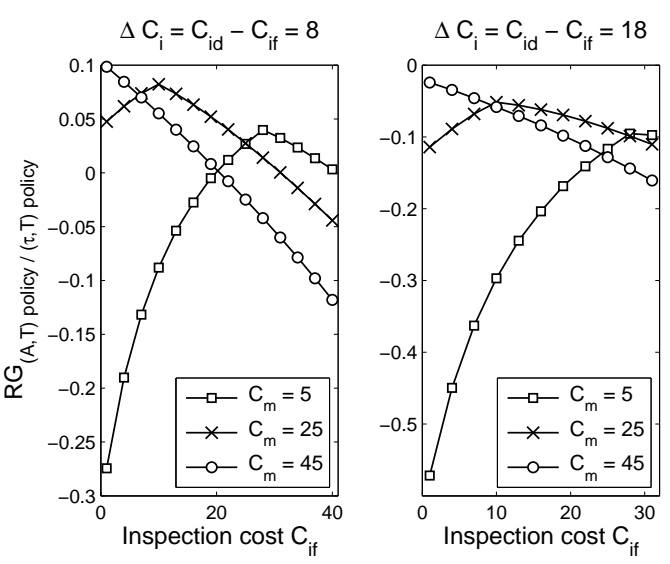

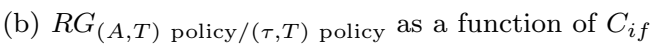

Figure 8: Relative gain on the optimal maintenance cost rate of the $(A, T)$ policy compared to the $(\tau, T)$ policy for different minimal repair costs

on the characteristics of the maintained system, and it is a compromise between a loss due to a more expensive inspection and a gain due to the minimal repair decision at the right time according to the system condition. Notwithstanding the above, we can still bring out some conclusions for some observed cases. For example when $\Delta C_{i}$ and $C_{i f}$ are small enough and the variance in the degradation increment is large, the $(A, T)$ policy is more profitable than the $(\tau, T)$ policy for (see figure 7$)$. This is because the minimal repair under the $(A, T)$ policy is a condition-based decision which can adapt to the real system state better than an age-based minimal repair decision in $(\tau, T)$ policy, and especially with a high variance of the degradation 
process. Under this situation, it is indeed useful to follow closely the actual evolution of the degradation path to carry out a condition-based minimal repair instead of a "blind" time-based minimal repair. Figure 8 also shows some situations where depending on the minimal repair cost and inspection cost, the $(A, T)$ policy can give more saving in maintenance cost when compared to the $(\tau, T)$.

Consequently, through the numerical results analyzed in subsections 4.2.1, 4.2.2 and 4.2.3, we can conclude that the minimal repair action can be more profitable than a system replacement when the inspection and the minimal repair are not too expensive, especially for a repairable system with a high shock failure rate and a low degradation speed. The different approaches of minimal repair decisions should be carefully used according to the different characteristics of a system to avoid inopportune maintenance spending. When the difference of inspection cost between both minimal repairs approaches is high, and the system degradation process is quite smooth (low variance in the degradation increment), the use of time-based minimal repair approach can be more adequate than condition-based minimal repair approach. Otherwise, using the condition-based minimal repair approach can lead to a significant saving in the maintenance cost. Notwithstanding the above, an analysis on the relative gain in the maintenance cost among the maintenance policies should be used before choosing a maintenance policy.

\section{Conclusions}

The present paper provides a realistic extension of the DTS model by introducing an interdependence of the shock and degradation processes for a single-unit repairable system. The analytical formulation of expected cost rate for the two proposed age-based maintenance policies (i.e. the policy implementing timebased minimal repairs and the policy implementing condition-based minimal repairs) has been obtained. The comparisons of the relative gain in the optimal maintenance costs for both maintenance policies under different system characteristics have been made. Using numerical examples, it has been shown a minimal repair can lead to a substantial saving in maintenance cost. However one has to be cautious when deciding the approach of minimal repair actions: an analysis of the relative saving in the maintenance cost can allow one to make a right choice.

The present paper assumes that the degradation-based failure mode occurs when the degradation level of the system exceeds a fixed deterministic value $L$. However, in practice, depending on the operating environments, or on the use made by each customer, this failure threshold cannot be deterministic. Future research can be continued considering a model with a random degradation failure threshold. The uncertainty in the inspections could then be taken into account to make the model more realistic. 


\section{Acknowledgements}

For K.T. Huynh this work is part of his $\mathrm{PhD}$ research work financially supported by Conseil Régional de Champagne-Ardernne, France.

For I.T. Castro this research was supported by the Ministerio de Ciencia e Innovación, Spain, under grant MTM2009-07634. The work was carried out while I.T. Castro was visiting the University of Technology of Troyes in November 2009. She would like to thank the University of Technology of Troyes for making her visit possible.

[1] A. Jeang, "Tool replacement policy for probabilistic tool life and random wear process," Quality and Reliability Engineering International, vol. 15, no. 3, pp. 205-212, 1999.

[2] J. Van Noortwijk and H. Klatter, "Optimal inspection decisions for the block mats of the eastern-scheldt barrier," Reliability Engineering \&3 System Safety, vol. 65, no. 3, pp. 203-211, 1999.

[3] W.J. Hopp, Y.L Kuo, "An optimal structured policy for maintenance of partially observable aircraft engine components," Naval Research Logistics, vol. 45,pp. 335-352,1998.

[4] H. Hong, "Inspection and maintenance planning of pipeline under external corrosion considering generation of new defects," Structural Safety, vol. 21, no. 3, pp. 203-222, 1999.

[5] A. Lehmann, "Joint modeling of degradation and failure time data," Journal of Statistical Planning and Inference, vol. 139, no. 5, pp. 1693-1706, 2009.

[6] M. Pandey, X. Yuan, and J. van Noortwijk, "Gamma process model for reliability analysis and replacement of aging structural components," in ICOSSAR 2005, pp. 2439-2444, 2005.

[7] J. Van Noortwijk, "Optimal replacement decisions for structures under stochastic deterioration," in Proceedings of the Eighth IFIP WG, vol. 7, pp. 273-280, 1998.

[8] C. Barker and M. Newby, "Optimal non-periodic inspection for a multivariate degradation model," Reliability Engineering \&3 System Safety, vol. 94, no. 1, pp. 33-43, 2009.

[9] C. Bérenguer, A. Grall, L. Dieulle, and M. Roussignol, "Maintenance policy for a continuously monitored deteriorating system," Probability in the Engineering and Informational Sciences, vol. 17, no. 2, pp. 235$250,2003$.

[10] A. Grall, L. Dieulle, C. Bérenguer, and M. Roussignol, "Continuous-time predictive-maintenance scheduling for a deteriorating system," IEEE Transactions on Reliability, vol. 51, no. 2, pp. 141-150, 2002.

[11] N. Singpurwalla, "Survival in dynamic environments," Statistical Science, vol. 10, no. 1, pp. 86-103, 1995.

[12] A. Lemoine and M. Wenocur, "On failure modeling," Naval Research Logistics Quarterly, vol. 32, no. 3, pp. 497-508, 1985. 
[13] E. Deloux, B. Castanier, and C. Bérenguer, "Predictive maintenance policy for a gradually deteriorating system subject to stress," Reliability Engineering $\&$ System Safety, vol. 94, no. 2, pp. 418-431, 2009.

[14] W. Li and H. Pham, "An inspection-maintenance model for systems with multiple competing processes," IEEE Transactions on Reliability, vol. 54, no. 2, 2005.

[15] J. Van Noortwijk, J. Van der Weide, M. Kallen, and M. Pandey, "Gamma processes and peaks-overthreshold distributions for time-dependent reliability," Reliability Engineering \& System Safety, vol. 92, no. 12 , pp. 1651-1658, 2007.

[16] R. Barlow and L. Hunter, "Optimum preventive maintenance policies," Operations Research, vol. 8, no. 1, pp. 90-100, 1960.

[17] T. Nakagawa, "A summary of periodic replacement with minimal repair at failure," Journal of the Operations Research Society of Japan, vol. 24, no. 3, pp. 213-227, 1981.

[18] M. Finkelstein, "Some notes on two types of minimal repair," Advances in Applied Probability, vol. 24, no. 1, pp. 226-228, 1992.

[19] F. Beichelt, "A unifying treatment of replacement policies with minimal repair," Naval Research Logistics, vol. 40, no. 1, pp. 51-67, 1993.

[20] T. Aven and U. Jensen, "A general minimal repair model," Journal of Applied Probability, vol. 37, no. 1 , pp. 187-197, 2000.

[21] R. Zequeira and C. Bérenguer, "Periodic imperfect preventive maintenance with two categories of competing failure modes," Reliability Engineering $\&$ System Safety, vol. 91, no. 4, pp. 460-468, 2006.

[22] D. Bocchetti, M. Giorgio, M. Guida, and G. Pulcini, "A competing risk model for the reliability of cylinder liners in marine diesel engines," Reliability Engineering 6 System Safety, vol. 94, no. 8, pp. 1299-1307, 2009.

[23] C. Blain, A. Barros, A. Grall, and Y. Lefebvre, "Modelling of stress corrosion cracking with stochastic processes-application to steam generators," in Risk, Reliability and Societal Safety, Proceedings of the European Safety and Reliability Conference, pp. 25-27, 2007.

[24] E. Cinlar, E. Osman, and Z. Bazoant, "Stochastic process for extrapolating concrete creep," Journal of the Engineering Mechanics Division, vol. 103, no. 6, pp. 1069-1088, 1977.

[25] D. Frangopol, M. Kallen, and J. Van Noortwijk, "Probabilistic models for life-cycle performance of deteriorating structures: review and future directions," Progress in Structural Engineering and Materials, vol. 6, no. 4, pp. 197-212, 2004.

[26] M. Abdel-Hameed, "A gamma wear process," IEEE transactions on Reliability, vol. 24, no. 2, pp. 152$153,1975$.

[27] J. Van Noortwijk, "A survey of the application of gamma processes in maintenance," Reliability Engineering $\&$ System Safety, vol. 94, no. 1, pp. 2-21, 2009.

[28] R. Nicolai, Maintenance models for systems subject to measurable deterioration. PhD thesis, Erasmus 
University Rotterdam - Timbergen Institude, The Netherlands.

[29] X. Yuan, Stochastic modeling of deterioration in nuclear power plant components. PhD thesis, University of Waterloo, Ontario, Canada.

[30] K.T. Huynh, A. Barros, C. Bérenguer, and I.T. Castro, "A periodic inspection and replacement policy for systems subject to competing failure modes due to degradation and traumatic events," vol. 96, no. 4,pp. 497-508, 2010.

[31] V. Gnedenko B.V., Korolev, Random summation: limit theorems and applications. CRC Press, 1996.

[32] H. Tijms, A first course in stochastic models, John Wiley and Sons, Inc. Wiley, New York, 2003.

[33] K.T. Huynh, A. Barros, C. Bérenguer, and I.T. Castro, "Value of condition monitoring information for maintenance decision-making," in Proc. Ann. Reliability $\&$ Maintainability Symp, pp. 543-548, 2010.

[34] L. Mann, A. Saxena, and G. Knapp, "Statistical-based or condition-based preventive maintenance," Journal of Quality in Maintenance Engineering, vol. 1, no. 1, pp. 46-59, 1995.

[35] M. Rausand and A. Høyland, System reliability theory: models, statistical methods, and applications. Wiley New York, 2004.

[36] M. Berman, "Inhomogeneous and modulated gamma process," Biometrica, vol. 68, no. 1, pp. 143-52, 1981.

[37] K. Muralidharan, "A review of repairable systems and point process models," ProbStat Forum, vol. 01, pp. 26-49, 2008.

[38] R.E Barlow, F. Proschan "Mathematical Theory of Reliability," Classics in Applied Mathematics, SIAM, vol. 17,1996 .

[39] N. Singpurwalla, "Gamma processes and their generalizations: an overview" In R. Cooke, M. Mendel, and H. Vrijling, editors, Engineering Probabilistic Design and Maintenance for Flood Protection, vol. 01, pp. 67-75, Dordrecht: Kluwer Academic Publishers,1997.

[40] R.A. Dagg, "Optimal Inspections and Maintenance for stochastically deteriorating systems," $P h D$ thesis, The city University, London,1999.

[41] W.J. Hopp, S.C Wu, "Multiaction Maintenance under Markovian Deterioration and Incomplete State Information," Naval Research Logistics, vol. 35,pp. 447-462,1988.

[42] W. Stadje, D. Zuckerman, "Optimal Maintenance Strategies for Repairable Systems with General Degree of Repair," Journal of Applied Probability, vol. 28,pp. 384-396,1991.

[43] R.M. Feldman, "Optimal Replacement With Semi-Markov Shock Models", Journal of Applied Probabbility,vol. 13,pp. 108-117, 1976.

[44] E.P.C Kao, "Optimal replacement rules when changes of states are semi-markovian," Operations Research, vol. 21,pp. 1231-1249,1973.

[45] P. Koselar, "Minimal cost replacement under markovian deterioration," Operations Research, vol. 12, no.9 pp. $694-706,1966$. 
[46] C.T. Lam, R.H. Yeh, "Optimal replacement policies for deteriorating systems under various maintenance strategies," IEEE Transactions on Reliability, vol. 43,pp. 423-430,1994.

[47] K.S. So, "Optimality of control limit policies in replacement models," Naval Research Logistics, vol. 39,pp. 685-697,1992.

[48] H. Wang, "A survey of maintenance policies of deterioration systems - Invited Review" European Journal of Operational Research,vol. 139, pp. 469-489, 2002.

[49] T . Nakagawa, "Maintenance theory of reliability" Springer Series in Reliability Engineering,Springer, 2005. 